\title{
On the nature of the photon and the electron
}

\author{
J.G.Williamson ${ }^{a}$ \\ ${ }^{a}$ University of Glasgow, College of Science \& Engineering, Glasgow G12 8LT, Scotland;
}

\begin{abstract}
A new theory, describing both light and material particles, is proposed. The experimentally-observed nature of space and time are brought into the theory at the most fundamental level. An equation encompassing the usual free-space Maxwell equations but similar in form to the Dirac equation is proposed. This equation has new kinds of solutions. Propagating, pure-field solutions may have any energy, but the energy transferred must be proportional to the frequency. These are identified with the physical photon. Solutions with a rest-mass term allow any incoming propagating field to merge into re-circulating vortex-like solutions. The minimum energy configuration "rectifies" the oscillating electric field of light into a uni-directional, radial (inward or outward directed) configuration. The resulting apparent external charge may be readily estimated and is found to be of the order of the elementary charge. The spin may, likewise, be calculated, and is found to be half integral, exhibiting a double-covering internal symmetry. Charge is then not a fundamental quantity in the theory - but is a result of the way field folds from a rest-massless bosonic to a rest-massive fermionic configuration. The simplest such charged, fermionic particles are identified with the electron and positron.
\end{abstract}

Keywords: Electromagnetic electron positron photon

\section{INTRODUCTION}

A companion paper has outlined many aspects of the theoretical basis to be used here. ${ }^{1}$ Space-time, at its simplest, is described by four and only four "linear" degrees of freedom. Within this space-time exists energy encapsulated in light and material particles: photons, leptons and hadrons. It has been fashionable in science, for the past half-century or so, to take complicated starting positions involving an extensive a-priori mathematical and conceptual structure to describe these objects. Complex groups have been taken as the starting points of many theories too numerous to mention. Further, there has been a tendency to wish to "quantise" everything from the beginning. It should seem self-evident that, in doing that, one loses the possibility of finding out why such things may be quantised at all. Putting in a quantisation or a symmetry observed experimentally as a starting axiom has some merit of course, but is a poor starting choice if one wants to understand the origins of that quantisation or symmetry. In this paper an attempt will be made to avoid any superfluous complexity, keep everything possible continuous and linear and keep the a-priori basis as simple as possible. Accordingly, all that will be introduced are four vector basis "directions" in space and time, their properties under multiplication and division, and a rest mass-energy term. This rest-mass energy is introduced, as described in the companion paper, ${ }^{1}$ as a square-root energy density denoted here "vot". The paper will study how this vot may flow between fundamental underlying space-time forms. A set of new linear differential equations equations are developed which encompass Newton's laws and the Maxwell equations. These new equations have re-circulating field and rest-massive solutions corresponding to four states: spin "up" and spin "down" electrons and positrons. These are proposed as physical Dirac spinors. ${ }^{2}$ This justifies making the association between vot as manifested in various space-time forms and physical quantities such as rest-mass (scalar vot), charge and current (vector vot), field (bi-vector vot) and angular momentum (tri-vector vot). The new theory allows certain quantities usually viewed as fundamental to be calculated from others. For example, the elementary charge $q$ may be calculated from the elementary angular momentum $\hbar$, or vice-versa.

Further author information: (Send correspondence to J.G. Williamson)

J.G. Williamson: E-mail: john.williamson@glasgow.ac.uk, Telephone: +44 1413304923 


\section{THE BASIS OF SPACE AND TIME}

Four unit vector components $\alpha_{0}, \alpha_{1}, \alpha_{2}, \alpha_{3}$ representing one temporal and three spatial unit lines respectively are introduced. These are labelled $\alpha_{0}$ for time, and $\alpha_{1}, \alpha_{2}$ and $\alpha_{3}$ for three perpendicular directions in space. These latter three may conveniently be thought of as being the unit " $x$ ", " $y$ " and " $z$ " of Cartesian space. The Maxwell equations (and any other linear equations) are, however, equally valid in any proper, conformal, orthonormal system of co-ordinates. As is well-known, if solutions exist in any such space, there also exists corresponding solutions in any other such space. ${ }^{3}$ Here we will use the right-handed ordered triple $(1,2,3)$ for the general case, $(x, y, z)$ for Cartesian, $(r, \phi, z)$ for cylindrical, $(r, \theta, \phi)$ for spherical and $(\rho, \theta, \phi)$ for toroidal co-ordinates.

Under strict definitions ${ }^{1,2}$ of "multiplication" and "division" these lead to self-consistent relativistic properties of further unit elements derived from them at any level of expansion and to any differential order. Because the space-time basis has four linearly-independent base unit elements, under multiplication twelve further such unit geometrical objects are generated.This leads to sixteen linearly independent units elements in all. This may seem at first complicated, even larger than the 11 dimensions in some string theories, but these unit objects are quite simple, most of them being quite familiar to a child of three. They are just such things as unit planes and volumes, with only the unit scalar (point), quadrivector (4-volume) and spatio-temporal planes requiring much more thought and mathematical explanation.

It is neither desirable nor necessary to introduce a-priori any group on these other than that implicit in the physical algebra of points, lines, planes, volumes and a 4-volume. In the usual parlance of the field one might talk of introducing the Lorentz group at this point (but perhaps not the Poincaré group - since general translations lie outside the allowed light-cone of the restrictions of absolute relativity). The closest match would then be to introduce the Clifford group corresponding to the space-time algebra $\mathrm{Cl}(1,3),{ }^{4,5}$ but that is not a division algebra ${ }^{1}$ which is widely thought to cause problems. ${ }^{6}$ Imposing any of these a-priori, however reasonable they may appear, may introduce deep-held convictions of how a well-behaved mathematics should behave. Such convictions may, or may not, be paralleled in nature. Mathematics is a powerful language which allows one to think things that could otherwise not be thought at all. Its misuse, however, may render one blind to aspects of experimental reality which should otherwise be blindingly obvious.

An algebra may be designed strictly simpler than if the general consensus of the meaning of a "group" were to be imposed. The intention here is to keep the basis of the mathematics as minimal as possible. This will mean that, for example "multiplication" and "division" will be defined for the individual unit elements but "addition" and "subtraction" will not. That this is necessary should be self-evident: there is no such thing as a double-sized unit element. This is not to say that addition is not important. It is crucially and fundamentally important. Indeed addition is so important that it will be forced here to apply simultaneously to more than one kind of quantity: both the total integral energy and the local square-root energy density manifesting in many different space-time forms. The reason for forcing this on the mathematics is simply experimental: energies must add linearly but other quantities such as fields, for example, (corresponding to a square-root density of energy) are also observed to add linearly. Addition is implemented, not in the unit elements, but in the square-root density energy represented by a real-number factor. It turns out that, to achieve this dual linearity of energy and field, it is space and time themselves which must transform from frame to frame and locality to locality. The transformation required is just that of special relativity, as has been shown in the companion paper to this one. ${ }^{1}$ To aid readability in the following a shortcut will sometimes be used, with "square-root energy density" being replaced with the short form "root-energy" where the "square" and the "density" should be understood.

It should be taken here that, if a particular process is not allowed in the mathematical system, then it must be taken to be forbidden. A discussion of how this proscribes any logical system has been given by Wittgenstein. ${ }^{7}$ If the reader finds keeping in mind this kind of separation of the areas to which addition, multiplication and division should be applied too uncomfortable, read no further. The judgement of whether this kind of distinction has had any value must be considered in retrospect.

To introduce the proper (Lorentz-like) properties of space and time, multiplication of the temporal component $\alpha_{0}$ by itself is defined such that its square yields a fundamental positive (Lorentz) invariant scalar unity $\alpha_{0}^{2}=\alpha_{P}$. For this to be consistent with relativity (and experiment: the square of 4-vectors are Lorentz invariants) the three spatial components $\alpha_{1}, \alpha_{2}$ and $\alpha_{3}$ must then square to the negative scalar unity $\alpha_{i}^{2}=-\alpha_{P}$. Here and in the 
sequel, Greek indices run from 0 to 3 and Roman from 1 to 3, with 0 representing the time "direction". That is, it is taken that the square (or quotient) of the base line elements with themselves yields a unit invariant "point" element. This is a point as opposed to a line or a plane, not in the common meaning of an infinitesimal place. In English the word "point" is often used to denote a place with zero extent - sometimes, perhaps incorrectly, called a mathematical point. Such points are (usually) frame dependent. Here, it is considered that a place, for any given observer, is better defined by a relative position vector with respect to that observer. This is a line vector. Vectors are not invariant under general Lorentz rotations: a Lorentz boost will convert elements of time into elements of space and vice-versa. The unit point $\alpha_{P}$ is invariant under such transformations. It is the author's view that a physical point, especially the point corresponding to the point of view of an observer, is better defined with respect to its property of invariance under (general) Lorentz rotations than as the limit of a line (or volume) tending to zero length (or capacity). The unit scalar point $\alpha_{P}$ is not taken to be a place or an event, it is simply that frame-independent unit sized element invariant under a general Lorentz rotation or boost (and also under several other transformations such as an order inversion). It must be clearly distinguished from the other unit scalar here, the real number 1, which represents an amount or extent and not a fundamental space-time form.

In previous work the root rest-mass density component, which takes the geometrical form $\alpha_{P}$ in the new theory, has been called the pivot. ${ }^{2,8}$ To make a connection with the earlier work, the new word "vot" has been invented to express square-root mass-energy density in any space-time form. Many particles end with the suffix -on, this new concept is designed to express an underlying nature (of energy) even more elementary than that of particles - square-root energy density as conditioned by the space-time nature of elementary forms such as lines, planes and volumes. This root-energy form will be argued here, indeed to give rise to the inner physical properties of the photon and the electron and is hence considered here to be more "fundamental" than either. The new word vot may be used alone or as a suffix and the nomenclature for the scalar, rest-mass density here will be the p-vot. The closest existing conceptual description to an element of p-vot is to think of it as a square-root mass-energy density. The p-vot is then similar in some ways to the probability amplitude (wavefunction) in non-relativistic quantum mechanics often denoted $\Psi$. To derive the energy, $\Psi$ must be multiplied by an appropriate constant ( $\sqrt{J}$ for energy), squared and integrated over a relevant volume. It is usual to associate other quantities with this, such as the charge density, and the extended charge distribution of individual electrons may be readily measured experimentally. ${ }^{9}$ Here charge will arise, not as a fluid associated with mass, but as a coherent property of p-vot and field. Anything taking the same geometrical form as the p-vot is strictly always invariant under a Lorentz transformation; the symbol " $\Psi$ " and the concept of a wave-function are often used in the literature (perhaps confusingly) to describe things which are not. The p-vot may take, just as is the case for any field or density component, any magnitude. This is then an invariant scalar "point" quantity that can have any size (denoted by a real number prefix). Given that this quantity has similar properties with respect to energy to those of the electromagnetic field or probability amplitude, where the energy is derived by squaring and integrating over an appropriate volume, p-vot may be conveniently thought of as a square-root rest mass field. In the locality of matter, this mass-field will be strongly scavenged and confined by existing material particles appearing as low-energy heat. It may, however, be exchanged between particles by intermediating photons, as is usual in quantum electrodynamics.

Considering the important measurable properties of electromagnetism, Maxwell ${ }^{10}$ identified three pairs of quantities whose product had the dimensions of an energy density: the "electrostatic pair" (the electric field $\mathscr{E}$ and the electric displacement $\mathscr{D}$ ), the magnetic pair (the magnetic flux $\mathscr{B}$ and the magnetic field $\mathscr{H}$ ) and the "electrokinetic pair" ( the vector potential $\mathscr{A}$ and the current density $\mathscr{J}$ ). Note that, for Maxwell, the relationship between vector potential and current was similar to that between field and flux or displacement in that they were taken to represent the same underlying physical quantity in free-space, differing only by their units. The energy density for the electric field in S.I. units is: $\frac{1}{2} \mathscr{E} \mathscr{D}=\frac{1}{2} \epsilon_{0} \mathscr{E}^{2}$ and for the magnetic field it is $\frac{1}{2} \mathscr{B} \mathscr{H}=\frac{1}{2} \epsilon_{0} c^{2} \mathscr{B}^{2}$, where $\epsilon_{0}$ is the electric constant and $c$ is the speed of light respectively. Similarly, for the 4-potential $\mathscr{A}$ (units V.s. $m^{-1}$ ) and the 4-current $\mathscr{J}$ (units $A . m^{-2}$ ) the product $\mathscr{A} \mathscr{J}$ represents an energy density in $J \mathrm{~m}^{-3}$. Though all of these products represent energy densities, it is important to note that they are not all independent, being derived from each other by linear equations. To avoid double counting care must be taken as described by Maxwell ${ }^{10}$ and discussed more recently by Mead. ${ }^{11}$ If one thinks in terms of the p-vot, a square-root mass density invariant under a Lorentz transformation, the natural units would be $\sqrt{\left(\mathrm{kg} \mathrm{m}^{-3}\right)}$. For 
the electric field they would be $V m^{-1}$, for the electric displacement $\mathrm{Cm}^{-2}$, Tesla for the magnetic flux, $A m^{-1}$ for the magnetic field. More generally, in terms of energy, the units $\sqrt{\left(J \mathrm{~m}^{-3}\right)}$ are used here, and spatial units are also expressed in seconds, as described in the companion paper. ${ }^{1}$ The plethora of units reflects, amongst other things, the desire to understand the interaction of light with material systems. This is, in itself, a worthy aim. Here, one is seeking to describe how the elementary particles, which themselves make up material systems, might come into being. To keep the initial step for this simple enough to have any hope of understanding, the mathematical system is kept as simple as possible - partly by allowing only one kind of "field". Here the "electric field" is defined as $E \equiv \sqrt{\mathscr{E} . \mathscr{D}}$ and the "magnetic field" as $B \equiv \sqrt{\mathscr{B} . \mathscr{H}}$. One can always re-introduce the distinctions, complexity and beauty of the usual electric displacement $\mathscr{D}$, the electric field $\mathscr{E}$, the magnetic flux $\mathscr{B}$ and the magnetic field $\mathscr{H}$ at a later stage by using the constitutive relations in the usual way.

The algebra is defined such that anything with the geometrical form of a vector, in the directions of $\alpha_{0}, \alpha_{1}, \alpha_{2}$ and $\alpha_{3}$, transforms as a 4 -vector. Root-energy of this form is denoted here v-vot. Six unit "areas", properly denoted bi-vectors, are derived from the base lines by multiplying mutually perpendicular pairs such that unit plane element is, for example, $\alpha_{1} \alpha_{2}=\alpha_{12}$. Because there are four unit directions, there are six such planes: $\alpha_{23}, \alpha_{31}, \alpha_{12}, \alpha_{10}, \alpha_{20}, \alpha_{30}$. Root-energy here is then b-vot. The algebra forces these to transform relativistically as do the $(1,2,3$,$) components of the magnetic (B)$ and electric $(E)$ field respectively. ${ }^{12}$ Because of this, the six components of field with this form may also be denoted f-vot. If desired, the three components of the electric field could be denoted E-vot and those of the magnetic field B-vot, but this is a frame-dependent choice as under a Lorentz transformation elements of the electric field transform to the magnetic and vice-versa. Note that, if one allows, conceptually, one and only one bi-vector quantity in the mathematics, b-vot=f-vot. This would mean that, for extensions to the theory, the electric field term may mix in with the momentum density term as both are space-time bi-vectors. This possibility will be explored in the section on new electron-like solutions below. Alternatively, one could distinguish the momentum-like quantities as m-vot, but this would enlarge the algebra and will not be followed here, but reserved for future work. The 4 tri-vectors (the dual of the vectors) represent unit "volume" elements $\left(\alpha_{123}, \alpha_{023}, \alpha_{031}, \alpha_{012}\right)$. The latter three are a momentum density multiplied by a perpendicular unit vector, and therefore transform as the components of an angular momentum density. These are denoted t-vot. Finally, there is a single quadri-vector, $\left(\alpha_{0123}\right)$ which, just as the scalar, is invariant under a Lorentz transformation but, similarly to the unit imaginary $i$, squares to the negative unity $\alpha_{0123}^{2}=-\alpha_{P}$. Square-root energy with this form would be denoted q-vot. In total, there are then sixteen linearly independent space-time forms. Six square to the positive scalar unity $\alpha_{P}: \alpha_{0}, \alpha_{10}, \alpha_{20}, \alpha_{30}, \alpha_{123}$ and $\alpha_{P}$ itself. The remaining ten square to the negative scalar unity $-\alpha_{P}: \alpha_{1}, \alpha_{2}, \alpha_{3}, \alpha_{23}, \alpha_{31}, \alpha_{12}, \alpha_{023}, \alpha_{031}, \alpha_{012}$ and $\alpha_{0123}$.

Using this kind of algebra proves to provide a better parallel of the physical way energy flows through space and time than do alternatives. It should be noted that the concept of "volume" is not frame-independent. This concept is taken care of by the proper transformations of space within the algebra. In any given frame and locality volume is well-defined and hence vot is conserved locally for each and every Lorentz frame. To keep track of this within the mathematics, the principle of absolute relativity is imposed here such that no quantity may appear without its proper geometrical form, as defined by one of the sixteen distinct unit elements outlined above. This has the crucial property that the proper local values of all quantities transform correctly between each other in every Lorentz frame. For those wishing to gain further insight into the properties of the sub-algebra than that expressed in the companion paper, the sub-algebra of the space-time unit elements amongst themselves parallels the Clifford algebra $C l(1,3)^{4}$ championed by Hestenes as the space-time algebra in internal models of the electron structure $^{5}$ and is a simplification, in some ways, of certain Dirac algebras. ${ }^{13}$ The closest algebra discussed in the literature is sometimes called the Clifford-Dirac algebra and has been well-described in recent popular books ${ }^{6}$ or more extensively in recent textbooks. ${ }^{4}$ As mentioned before, the precise algebra used here, however, has certain extra restrictions not usual in standard approaches with either. The reader is referred elsewhere for details. ${ }^{1,2,8,12}$ In this paper, the consequence of adding a seventh (and eventually eighth) term to the six components of the electromagnetic field in this formalism, corresponding to a dynamical scalar (or pseudo-scalar) rest-mass, will be discussed. The resultant effect on the electromagnetic field is to cause the momentum flow to deviate in the direction of the electric (or magnetic) field. A solution with radial electric field then allows a solution with a re-circulation of electromagnetic energy. An electromagnetic vortex in momentum space. The re-configuration of the field forces the electric field - oscillating in the photon, to become rectified in the sense that it becomes either radially inward-directed (electron-like) or outward directed (positron-like). ${ }^{14}$ Similarly, the fundamental 
spin, integral for the photon, is half-integral for the simplest such charged particle. The method and topology of the confinement forces pure-field to re-configure in the process of the creation of particle-antiparticle pairs, from uncharged photons with integral spin to particles of equal and opposite charge with half-integral spin. Conversely, the annihilation of particle-antiparticle systems to photons cancels the topological vortex-anti-vortex pair in the recirculating system - allowing the particle-antiparticle system to decay to two free photons. Both processes conserve both charge and angular momentum - not only globally, but also locally. The fact that halfintegral and integral spin arise naturally from the topologies of solutions and the inter-actions between them, explains why a mere magnitude should be correlated with such far-reaching effects as it is in the statistics of fermions and bosons.

\section{EXTENSION OF THE MAXWELL THEORY}

The unit elements give the proper form and the relativistic transformation properties of the vector and hence the scalar and the the bi- tri- and quadri- vectors, but to represent an actual quantity, these must also have a magnitude. For this real numbers are used - keeping an explicit separation between the amount (of a scalar energy for example) or magnitude (of a vector force for example) and its proper form. This is not merely a question of style, it is this rigour that allows the imposition of absolute relativity at all levels and that gives rise to the new results of light quantisation and the formation of charged fermions from uncharged bosons in the following.

A four vector is written $\left(\mathbf{v}=a_{0} \alpha_{0}+a_{1} \alpha_{1}+a_{2} \alpha_{2}+a_{3} \alpha_{3}\right)$. The $a_{\mu}$ are real number quantities expressing a magnitude (and the physical units) and the $\alpha_{\mu}$ are fundamental, invariant unit lines which may take at most the two values $\pm \alpha_{\mu}$. Here and in the sequel, Greek indices run from 0 to 3 and Roman from 1 to 3, with 0 representing the time "direction".

For Cartesian co-ordinates a 4-vector 4-differential is defined as:

$$
\begin{aligned}
\mathscr{D}_{4} & =\frac{\partial}{\alpha_{\mu} \partial x_{\mu}}=\partial_{\mu} / \alpha_{\mu} \\
& =\alpha_{0} \partial_{0}-\alpha_{1} \partial_{1}-\alpha_{2} \partial_{2}-\alpha_{3} \partial_{3}=\alpha_{0} \partial_{0}-\alpha_{i} \vec{\nabla}
\end{aligned}
$$

Where the subscript on the left denotes the number of distinct unit elements in the expression and in this case, denotes that it is a 4 -vector derivative. Note that the 4 -vector differential operator implicitly contains inverse vector unit elements: this is a requirement of the principle of absolute relativity. The over-arrow denotes a conventional three-vector, as in the original formulation of (the Helmholtz form of) the Maxwell equations. Note the change of sign of the space components due to the implicit quotient of the unit vectors and the fact that the three spatial vectors $\alpha_{1}, \alpha_{2}$ and $\alpha_{3}$ square to the negative scalar unity $\alpha_{i}^{2}=-\alpha_{P}$. The $x_{\mu}$ are taken here to have the same physical units. Expressing all quantities in seconds, for example means that $c x_{i}=t$. The differential above then has units $s^{-1}$ but may be made dimensionless by multiplying by an appropriate unit time $\mathcal{T}$, which may be taken to be the inverse Compton frequency for any physical system.

The 4-differential of a 4-vector (v-vot identified initially as a 4-vector potential) conventionally yields field components. Writing v-vot as:

$$
A_{4}=\alpha_{\mu} A_{\mu}=\alpha_{0} A_{0}+\alpha_{1} A_{1}+\alpha_{2} A_{2}+\alpha_{3} A_{3}=\alpha_{0} A_{0}+\alpha_{i} \vec{A}
$$

The $16(=1+3+3 \cdot 2+3 \cdot 2)$ terms of the 4 -derivative of the v-vot $\mathscr{D}_{4} A_{4}$ may then be gathered together and written as p-vot and b-vot components:

$$
\mathscr{D}_{4} A_{4}=\alpha_{P}\left(\partial_{0} A_{0}+\vec{\nabla} \cdot \vec{A}\right)-\alpha_{i 0}\left(\partial_{0} \vec{A}+\vec{\nabla} A_{0}\right)-\alpha_{i j} \vec{\nabla} \times \vec{A}=P \alpha_{P}+F \alpha_{\mu \nu}
$$

which is the sum of a scalar (pivot) part $P \alpha_{P}$ and a bivector (field) part $F \alpha_{\mu \nu}$. The second and third term are,conventionally the electric field and the magnetic field respectively. The first (scalar) term is a gauge term. If zero we have the Lorenz gauge, and zero rest mass. This is the proper gauge for light-like solutions. Setting this non-zero one has other gauges, but also, necessarily, non-zero rest mass. The extension including the 
corresponding 4-derivative of the 4-trivector potential, $\mathscr{D}_{4} T_{4}$, would yield additional field components as well as a quadri-vector term $Q \alpha_{0123}$.

The theory here will investigate simply the properties of the transformation of vot between different space-time forms. It will not allow two different quantities to represent the same physical field or current, in particular it will allow one and only one bi-vector quantity, the electromagnetic field, denoted $F$ and split into electric $(E \equiv \sqrt{\mathscr{E} \mathscr{D}})$ and magnetic $(B \equiv \sqrt{\mathscr{B} \mathscr{H}})$ parts. Similarly, the theory will allow one and only one vector quantity, which may be visualised as either the 4 -current density or the 4 -vector potential, denoted here $A \equiv \sqrt{\mathscr{J} \mathscr{A}}$. This is a significant departure from the conventional approach, and has consequences in fixing the gauge for example, which some may find uncomfortably restricting. The main advantage of this is that it will allow the derivation of the elementary charge from the flow of the mass-field and the electromagnetic field, rather than having to insert it a-priori. It must be judged whether the loss of some gauge freedom is worth an understanding of the underlying nature of charge, quantum spin, and an understanding of the underlying nature of both the photon and the electron. The approach here will be to develop this strongly-constrained theory, compare it with the Maxwell and Dirac theories, and investigate its consequences in terms of new solutions. The reader must then judge whether or not the resultant benefits outweigh the costs.

Over each of the sixteen multivector-quantities defined above, a general dynamical multi-vector field $G_{16}$ is defined over a scalar term $P$, a vector term $A_{4}$, a bi-vector field term $F_{6}=F \alpha_{\mu \nu}=E_{i} \alpha_{i 0}-B_{i} \alpha_{j k}$, a trivector term $T_{4}=\alpha_{123} T_{0}+\alpha_{023} T_{1}+\alpha_{031} T_{2}+\alpha_{012} T_{3}$ and an eventual quadri-vector term $Q$ such that: $G_{16}=$ $P \alpha_{P}+A_{0} \alpha_{0}+A_{i} \alpha_{i}+E_{i} \alpha_{i 0}-B_{i} \alpha_{j k}+T_{k} \alpha_{0 i j}+T_{0} \alpha_{123}+Q \alpha_{0123}$.

Writing, by analogy with the form of the free-space Maxwell equation $\mathscr{D}_{4} F_{6}=0, \mathscr{D}_{4} G_{16}=0$, and again using the conventional 3 -space patterns for reference, one obtains a more general equation:

$$
\begin{array}{r}
\mathscr{D}_{4} G_{16}=\alpha_{0}\left(\vec{\nabla} \cdot \vec{E}+\partial_{0} P\right)+\alpha_{123}\left(\vec{\nabla} \cdot \vec{B}+\partial_{0} Q\right)+\alpha_{i}\left(\vec{\nabla} \times \vec{B}-\partial_{0} \vec{E}-\vec{\nabla} P\right)+\alpha_{0 i j}\left(\vec{\nabla} \times \vec{E}+\partial_{0} \vec{B}+\vec{\nabla} Q\right)+ \\
\alpha_{P}\left(\vec{\nabla} \cdot \vec{A}+\partial_{0} A_{0}\right)+\alpha_{0123}\left(\vec{\nabla} \cdot \vec{T}+\partial_{0} T_{0}\right)+\alpha_{i 0}\left(\partial_{0} \vec{A}+\vec{\nabla} A_{0}+\vec{\nabla} \times \vec{T}\right)+\alpha_{j k}\left(\partial_{0} \vec{T}+\vec{\nabla} T_{0}-\vec{\nabla} \times \vec{A}\right)=0
\end{array}
$$

Since all unit elements are linearly independent, the meaning of the zero on the right is that each expression on the left is zero separately. With $P$ and $Q$ zero the first four terms then correspond exactly to the freespace Maxwell equations. The Maxwell equations, in the present context, may be expressed as four coupled differential equations over the 24 terms of the expression $\mathscr{D}_{4} F_{6}=0$. The second four terms express a similar set of constraints between current-like and angular momentum-like quantities. The full set may be viewed as eight coupled differential equations over the 64 terms of the expression $\mathscr{D}_{4} G_{16}=0$. Note that, in the extended theory, the first term implies that if the p-vot term $P$ is non-zero the electric field divergence (the charge) may be also non-zero and is proportional to the rate of exchange of mass-energy as in quantum electrodynamics. Also the (vector) current term contains an extra component over and above the displacement current introduced by Maxwell. ${ }^{10}$

Within the extended equations, the physical effect of the new scalar invariant mass term $P$, for example, is to curve the momentum transport direction in the direction of the electric field. If both are non-zero, this leads to the possibilities of a pivoting of the field flow around the resultant mass leading to new kinds of self-confined circulating solutions with rest-mass. ${ }^{2,17}$ Such a re-configuration (a rectification) of the field constitutues an element of charge, as has been argued in previous work ${ }^{14}$ and as will be discussed further below.

\section{NEW SOLUTIONS:THE PHOTON}

In the companion paper, a left circularly polarised electromagnetic wave, travelling in the the $+z$-direction in Cartesian space and transmitting a quantum of energy $\mathcal{E}$ in the centre of mass frame has been written:

$$
F_{L}=H_{0} U_{F} R \mathcal{E}\left(\alpha_{10}+\alpha_{31}\right) e^{\frac{\varepsilon}{\hbar} R\left(\alpha_{3} z-\alpha_{0} t\right) \alpha_{012}}=F_{0} R\left(\alpha_{10}+\alpha_{31}\right) e^{R\left(k \alpha_{3} z-\omega \alpha_{0} t\right) \alpha_{012}}=\mathcal{F W}
$$

This has a pre-factor part representing the initial (or final) field configuration $\mathcal{F}=F_{0} R\left(\alpha_{10}+\alpha_{31}\right.$ ), and a hypercomplex exponential wave-function $\mathcal{W}=e^{R\left(k \alpha_{3} z-\omega \alpha_{0} t\right) \alpha_{012}}$. The wave-part $\mathcal{W}$ has 4 parts: p-vot field, 
electric and magnetic fields (E-vot and B-vot parts) and a q-vot part. In the whole expression the rest-massive parts $P$ and $Q$ cancel identically and $\mathcal{F W}$, is a pure rest-massless field.

This wave-function $\mathcal{W}$ is not a solution itself of the free-space Maxwell equations but is a solution of the more general set of equations above such that $\mathscr{D}_{4} \mathcal{W}=0$. In the product $F_{L}=\mathcal{F} \mathcal{W}$, the (rest-massive) p-vot and q-vot terms cancel if and only if the field components conform to that which is observed physically (as written above), so that the whole expression is then a solution of the free-space Maxwell equations alone $\mathscr{D}_{4} F_{L}=0$. What this means, physically, is that, if $\mathcal{W}$ is an element of the proper relativistic wave-function of the emitter and absorber, such functions may couple to and propagate field configurations of arbitritary total energy. Such fields are rest-massless, and hence may be propagated at light-speed, only if the initial (emitter) and final (absorber) fields have equal and perpendicular magnetic field components and are such that the total energy in their frames (note carefully that these are usually different) is proportional to the local wave-function frequency $\nu$ such that $\mathcal{E}=h \nu$. This is exactly what is observed in experiment.

The real-number constants $c$ are the (scalar) speed of light and $\mathcal{E}(=\hbar c k=\hbar k=\hbar \omega=h \nu$ ) the (scalar) quantum of energy transmitted in the centre-of-momentum frame respectively. $U_{F}$ is a universal constant, converting to field units but dependent on the nature of the emitter and absorber. It takes the same value for all photons emitted and absorbed from systems quantised under the usual rules. The "usual rules" here includes the physics of most atoms, molecules and plasmas at anything much above a few Kelvin where transitions are between states of integral angular momentum. The quantisation of photons is not then a property of the field equations, which remain continuous, but of the properties of the emitter and absorber. The possibility that the quantisation may take other values under certain circumstances will be discussed in the section on "experimental tests". $H_{0}$ is a distribution function over the number of cycles in phase representing the spread of field over phase, whose square integrates to unity. This is an invariant and is the same in all frames, right up to the limit of light-speed where the integrated energy goes to zero. The single parameter $\mathrm{R}$ is that factor which determines the scales of energy, frequency, length and time. As discussed in the companion paper, ${ }^{1}$ the whole wave-function transforms properly under a general Lorentz transformation. In each of the frames appropriate to an "event" it is quantised in that it describes light coming in "lumps", with the energy transmitted proportional to the frequency. This function, though a major result in itself, has been discussed in more detail in the companion paper and is used, here, merely as the starting point for the development of the solutions to charged, material particles.

Equation (5) may be readily expanded in any particular frame. For the conditions corresponding to experimentally observed photons, the non field (scalar and quadri-vector) terms in the exponential part cancel. Setting $F_{1}=H_{0} U R \mathcal{E}$ and $k=\hbar c k=\omega=\frac{\mathcal{E}}{\hbar}$ one obtains:

$$
F_{L}=F_{1}\left[\left(\alpha_{10}+\alpha_{31}\right) \cos (k z-\omega t)+\left(\alpha_{23}-\alpha_{20}\right) \sin (k z-\omega t)\right]
$$

This describes electric $\left(\alpha_{i 0}\right)$ and magnetic $\left(\alpha_{i j}\right)$ fields rotating in time in a plane perpendicular to the direction of momentum transport and transforming in space from magnetic field to electric field and vice-versa. The resultant field configuration is that shown in figure 1. It appears identical to that found in any elementary textbook on electromagnetism for a left-handed circularly polarised wave. The wave-function in equation (5) describes a temporal rotation. This is not a rotation imposed by some external force, but is a natural consequence of forcefree motion as described by equation (5). This requires, nonetheless, that the lateral extent of any elements of rotation in the photon frame should not exceed a rotation horizon imposed by the speed of light. Note that this does not imply that the photon should rotate about a single axis. Such an axis is not defined by the equations. The only places where a physical photon is constrained spatially remain those of the emitter and absorber. The physical limitation of the rotation horizon, nonetheless, imposes conditions on the integral angular momentum of allowed solutions. The concept was used in previous work to lay bare the physical origin of the anomalous magnetic moment of the electron as a localised photon. ${ }^{14}$ For a given frequency, the limit imposed by the speed of light on rotation, the rotation horizon, is just $r_{h}=\frac{c}{\omega}$. Introducing the photon momentum observed in experiment, $\vec{p}=\hbar \omega / c$, gives a limit on the integral allowed angular momentum of the solutions of $r_{h} \times \vec{p}=\hbar$. This sets the intrinsic scale of unit angular momentum for all solutions such as that described by equation (5). In conclusion, demanding the principle of absolute relativity, manifested in the form of equation (5), places strong restrictions on allowed solutions, over and above those required by the Maxwell equations alone. Though the 


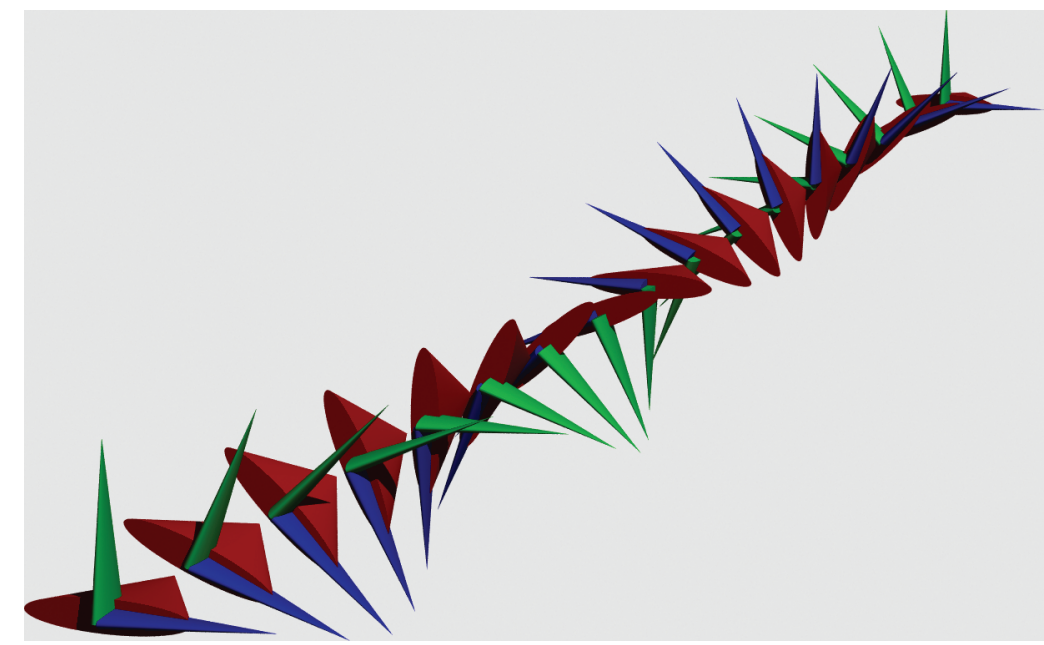

Figure 1. Representation of a single wavelength of a circularly polarised photon of equation (5). The electric field direction is represented using green arrowheads, the magnetic field blue and the momentum density (just $E \times B$ here) red.

underlying theory remains continuous, travelling wave solutions have energy proportional to frequency and a quantised angular momentum. This is the first result of this paper.

To understand why the Maxwell equations are, and always were, a sufficient condition for force-free motion of the constituent elements, consider a generalisation of the Lorentz force equation, the product of field and 4-current $F_{6} J_{4}$, long-considered by Einstein. ${ }^{15}$ Using the Maxwell equations to substitute for the 4-current $\mathscr{D}_{4} F_{6}=J_{4}$ and considering displacement currents alone in the pure-field case, one may generalise the Lorentz force equation to $F_{6}\left(\mathscr{D}_{4} F_{6}\right)=0$. Following the extended Maxwell, setting mass terms $P$ and $Q$ to zero (so considering field alone) and writing the electric and magnetic displacement currents from equation (4) as $\vec{J}=\alpha_{i}\left(\vec{\nabla} \times \vec{B}-\partial_{0} \vec{E}\right)$ and $\overrightarrow{J^{m}}=\alpha_{0 i j}\left(\vec{\nabla} \times \vec{E}+\partial_{0} \vec{B}\right)$ and, further, setting charge and magnetic monopole charge to zero one obtains for the generalised Lorentz force:

$$
\begin{aligned}
F_{6}\left(D_{4} F_{6}\right)= & \alpha_{0}\left(\vec{E} \cdot \vec{J}+\vec{B} \cdot \overrightarrow{J^{m}}\right)+\alpha_{123}\left(-\vec{B} \cdot \vec{J}+\vec{E} \cdot \overrightarrow{J^{m}}\right) \\
& +\alpha_{i}\left(-\vec{B} \times \vec{J}+\vec{E} \nabla \cdot \vec{E}+\vec{E} \times \overrightarrow{J^{m}}+\vec{B} \nabla \cdot \vec{B}\right) \\
+ & \alpha_{0 j k}\left(-\vec{E} \times \vec{J}-\vec{B} \nabla \cdot \vec{E}-\vec{B} \times \overrightarrow{J^{m}}+\vec{E} \nabla \cdot \vec{B}\right)
\end{aligned}
$$

Note that, for the magnetic field divergence and magnetic monopole current zero (as is usual), the vector term $\left(\alpha_{i}\right)$ is just the usual Lorentz force term. There are, however, other terms corresponding to "forces" without the vector form of a force. These act internally on the field. In this simple context force-free motion corresponds to $F_{6}\left(\mathscr{D}_{4} F_{6}\right)=0$. Note, however, for this to be zero it is sufficient that the free-space Maxwell equations are zero such that $\mathscr{D}_{4} F_{6}=0$. Anything satisfying the Maxwell equations, such as equation (5), is then a force-free motion of the electromagnetic field. Although there are (balanced) forces here, these are not sufficient by themselves to confine pure field to a localised solution such as that considered in earlier work. ${ }^{14}$ For that one must consider the further "forces" described by the 144 terms in the eight coupled non-linear equations derived from the product of the extend field and its derivatives such that $G_{16}\left(\mathscr{D}_{4} G_{16}\right)=0$.

\section{NEW SOLUTIONS: THE ELECTRON AND POSITRON}

A purely electromagnetic theory of the electron requires further forces capable of confining the electron charge, the so called Poincaré stresses.Within the new theory there is more than one way to look at the forces, at force-like terms and hence force-free motion. One may consider the generalisation of the Lorentz force and set this to zero in $G_{16}\left(\mathscr{D}_{4} G_{16}\right)=0$. Alternatively, one may generalise the expression for the 4-momentum density of the electromagnetic field $F F^{\dagger}=\frac{1}{2} \alpha_{P}\left(\vec{E}^{2}+\vec{B}^{2}\right)+\alpha_{i 0}(\vec{E} \times \vec{B})$ to the 4-momentum flow of a more general 


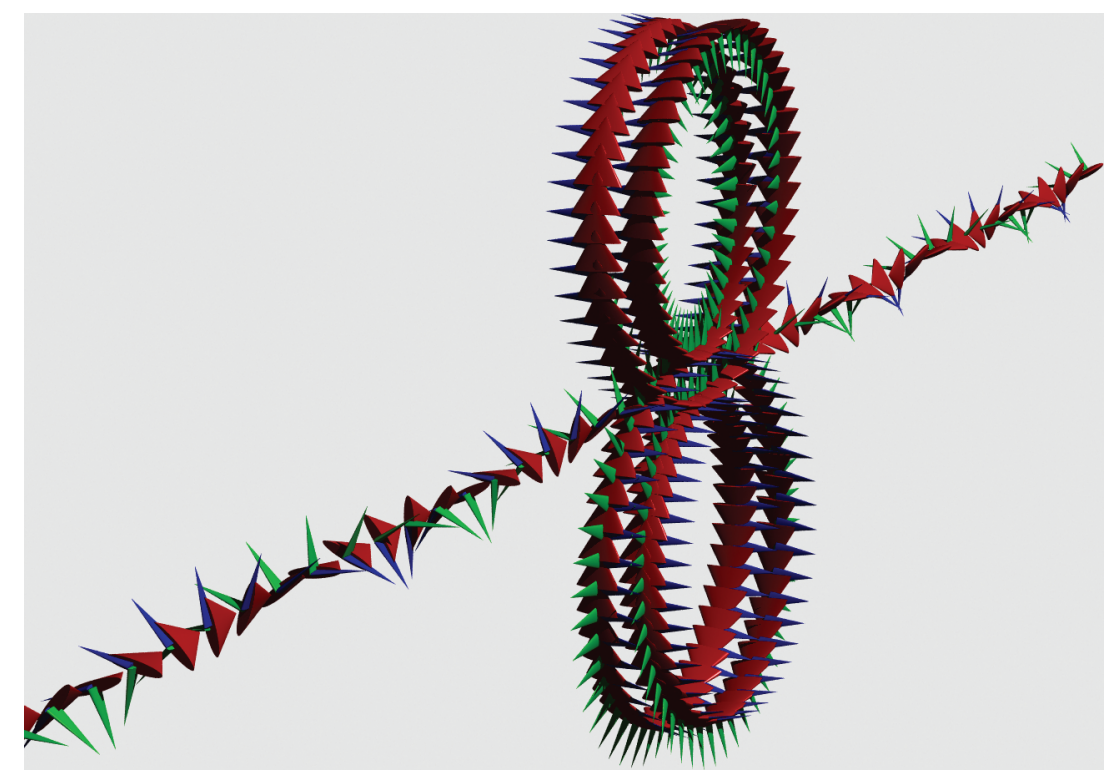

Figure 2. Diagram of the process of electron-positron pair creation or annihilation in phase (momentum) space at the point of separation. The incoming photons are shown on a different scale to the electron and positron vortices. The vortices are double-covering single wavelength loops with radius is a factor of $4 \pi$ smaller than the photon wavelength. The electron(above) encloses a pool of positive p-vot, the positron (below) a pool of negative p-vot.

field $G G^{\dagger}$ and develop an equation of motion for this (corresponding to Hamiltonian description or Newtons laws) by considering $\mathscr{D}_{4}\left(G G^{\dagger}\right)=0$. Both are sets of 144 coupled non-linear differential equations and both turn out to give similar, though not quite identical, results. They differ in that the change of signs engendered by changing the order of differentiation is slightly different to that in implementing the "Hermitian" conjugate within the sub-Clifford algebra, leading to a generalisation of the energy-momentum density and looking at the four differential of this quantity. In particular, both lead to the conventional expression for the Lorentz force, as outlined above, and both lead to internal "forces" with forms corresponding to space-space-space (volume) pressures and space-space-time "pressures". Both possible force equations have been considered in passing in earlier work..$^{8,17}$ Which, if either (or both!), corresponds with that observed in nature must be determined from experiment. Further, charged particles exchange photons with each other. This constitutes a further, pressurelike, force. A full consideration of the equations of motion of the resultant object must take all of these into account. It is beyond the scope of this paper to investigate the full implications of the extended linear equations $\mathscr{D}_{4} G_{16}=0$, let alone the non-linear generalised balanced force equations and external inter-action forces. Here, only the smallest possible extension to the theory of the six field components $F_{6}$ described by the free-space Maxwell equations to include a seventh component giving rise to an invariant (rest) mass, the p-vot $P$ will be investigated. That is one takes the subset $G_{16}=F_{6}+P$ and considers the equation $\mathscr{D}_{4}\left(F_{6}+P\right)=0$. It is not claimed here that this is necessarily the complete new equation governing the existence of and the full internal motion of the electron, that should involve more terms in the general equation and the interaction terms as discussed above. It does, however, introduce a new, essential feature into the theory of electromagnetism: a term allowing electro-pivot-magnetism to confine itself. Denoting the 7-component field and pivot as $G=F+P$ and the conjugate set as $G^{\dagger}=F^{\dagger}+P^{\dagger}$. The generalised energy-momentum density may then be written as:

$$
\frac{1}{2} G G^{\dagger}=\frac{1}{2}(F+P)\left(F^{\dagger}+P^{\dagger}\right)=\frac{1}{2} \alpha_{P}\left(\vec{E}^{2}+\vec{B}^{2}+P^{2}\right)+\alpha_{i 0}(\vec{E} \times \vec{B}+P \vec{E})
$$

It is apparent that, for the case $P=0$, one obtains the usual expression for the electromagnetic energy density $\frac{1}{2}\left(\vec{E}^{2}+\vec{B}^{2}\right)$ and for the momentum density (the Poynting vector) $\vec{E} \times \vec{B}$ as expected. The new feature for $P \neq 0$ is the emergence of an extra term in the rest mass-energy density $\left(\frac{1}{2} P^{2}\right)$ and an extra term in the momentum density $(P \vec{E})$. The effect of the $P \vec{E}$ term is to turn the direction of momentum propagation in the direction of 


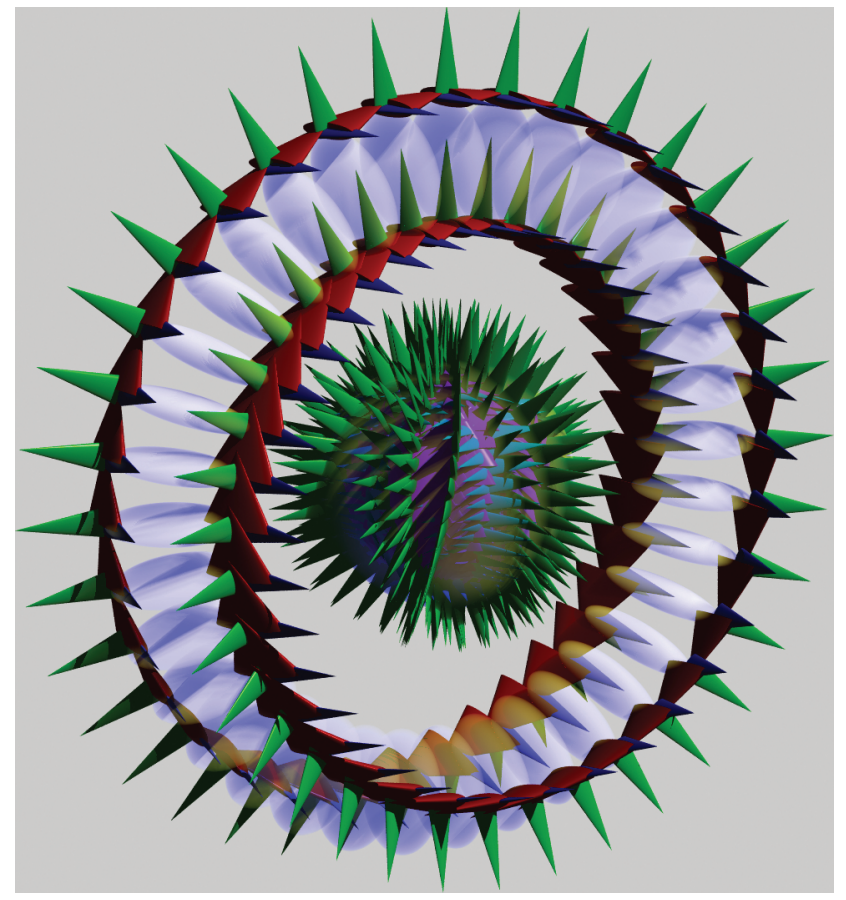

Figure 3. The toroidal field distribution in momentum space and the projection to the rest-massive sphere in normal space. This makes manifest both the internal double-loop and its relationship to the toroidal topology. The smoke disks representing p-vot in the momentum representation and correspond to slices through the hypersphere in space-time. These each project to the same sphere of p-vot. An artist's impression of what this would look like if one rotated at the same rate as the vortex is given in the core of the figure. Externally, one should observe a radial electric field and zero (on average) magnetic field.

the electric field. Force-free motion (the equation of motion) in this simple extension corresponds to the four differential of this being zero such that $\mathscr{D}_{4}\left(G G^{\dagger}\right)=0$. For a radial electric field this is a radial force allowing for spherically symmetric solutions. This allows the possibility of a closed vorticial flow in momentum space corresponding to elements of the field of a physical particle.

Electromagnetic flow in such a topology has been proposed in earlier work. ${ }^{14}$ It corresponds to an electromagnetic momentum flow around a toroidal topology in momentum space. The new feature in the present work is the interaction between p-vot and b-vot (mass and field), which leads to the possibility of a force-free re-circulating electromagnetic momentum with everywhere inward (or outward) directed electric field. Further, such an object, once it exists, could trap more field or emit part of it (subject,as usual, to energy-momentum conservation). This is a charged particle.

As mentioned in the introduction, a solution in Cartesian space such as equation (5) implies there exist similar solutions in any proper, conformal, orthonormal system of co-ordinates, modified only by well-known functions such as the half-integer Legendre polynomials. ${ }^{3}$ In particular, transposing equation (5) to toroidal co-ordinates $(\rho, \theta, \phi)$ "rectifies" the twisting oscillating field illustrated in figure 1 to be everywhere radial, as discussed in earlier work. ${ }^{14}$ This may be understood in terms of the twisting of the electromagnetic field about its momentum axis, as in equation (5) combined with a turning of this momentum direction in the direction of the electric field, as in equation (8). The twist and the turn then align to minimise the torsion and the field overlap. This occurs for a path for the field flow of equation (5) around a toroidal topology in momentum space. This minimum-energy configuration is that illustrated for the electron and positron in figure 2. The process of electron-positron decay into two photons (where the p-vot rest-mass terms in electron and positron annihilate), or the photo-production of an electron positron pair (where two equal and opposite pools of p-vot are created) may then be understood 
as a continuous process described by the new general linear equation (4). The electromagnetic field patterns in momentum space, in the process of the photo-production of an electron-positron vortex pair (or electron-positron annihilation), at the point where the electron and positron just separate (or where annihilation just begins) are illustrated in figure 2. For a complete separation to a free particle-antiparticle pair the initial photon energies must be sufficient to impart enough momentum to overcome the Coulomb attraction. In a stable solution, each of the elements of vot in the different space-time forms must transform between one another smoothly and locally such that each one is continuously replenished as it is destroyed, as described by the general equation (4). In this creation or annihilation process, all quantities, angular momentum, charge, current, local root-energy (and hence total integrated energy) must be locally conserved. The net result is that the zero-charge two boson initial state in two Cartesian topologies (the two photons) condenses into a zero charge final state in two toroidal topologies (the electron-positron pair) corresponding to two fermions with equal and opposite p-vot. Conversely, in pair annihilation the initial fermionic toroidal vortices (in momentum space) cancel to a bosonic Cartesian pair.

A single such particle, illustrating the essentially toroidal inner topology, is shown in figure 3 . The particle illustrated is the positron rather than the electron, since the outward-directed field shows the radial nature of the electric field more clearly. The smoke disks in the toroidal loop denote the pivot. The curvature illustrated is, however, not a curvature in ordinary space, but in momentum-space. This is because equation (8) is an equation in energy-momentum space not in space-space. It is a particular projection of the much more complication "motion" of the (potentially) sixteen linearly independent elements onto the bi-vector subset (and not the vector subset) of that space. It cannot be emphasised too strongly that the main subtlety to be taken on board in understanding the diagrams is that they are not drawn in ordinary space but in bi-vector space. This has, as described above, six degrees of freedom (six dimensions if you like). The form is toroidal in bi-vector space, but the projection to (the linearly independent three dimensions of) normal space is spherical. The curvature is the ratio between the $P \vec{E}$ term and the $\vec{E} \times \vec{B}$ term in equation (8). Though this has a physical value of $\frac{4 \pi}{\lambda_{C}} m^{-1}$, it is properly also a (space-space) bi-vector and not a vector term. In order to project on to a spatial distribution, it should be noted that, properly, each smoke disk represents a slice through a spherically symmetric p-vot sphere at the particle core. Since an isolated particle has nothing to rotate about but itself, all of these disks (representing spheres) should be drawn at the same point in space-time. Also the figure should rotate about a vertical axis such that the magnetic field is cancelled to some extent, leading to a minimal total energy. Experimentally, isolated electrons do not have a magnetic moment. Such a moment is induced only in an external field. This means that the field distribution shown should rotate, resonantly, at the Compton frequency, about a vertical axis - leading to an electric field radial in space - as is observed. Taking these together one arrives at a projection onto space like that illustrated in the core of the figure. A spherical ball of p-vot with a spherically symmetric, radial electric field. A charged particle.

\section{DISCUSSION}

The first question is: how can the initial state, with uncharged photons give rise to a final state with charged particles, an electron and a positron? Firstly, it should be noted that this is a process which occurs in nature, that is manifest in experiment. Charge is everywhere conserved in such a process. Particle-antiparticle pairs are created all over the place if there is sufficient energy. The author was lucky enough to work for many years at CERN - and there this kind of thing happens all the time. The primary process, if a high-energy photon or electron hits a material, is a shower of electrons, positrons and photons. If one's current favourite theory does not provide a satisfactory description of the details of this kind of process, then that theory is simply not good enough. It should, by the scientific method, be either refined or replaced with something that does.

The process illustrated in figure 2 is one where the field in the initial photon pair transforms, at least partially, into two equal and opposite pools of p-vot. These p-vot pools then deflect any incoming field into two counter-rotating vortices which curl up more and more tightly as more and more of the initial photon energy is incorporated into them. Charge is conserved in that the two final state particles (as well as the intermediate state vortices) have equal and opposite charge. Vorticity (spin) is conserved in that the vortices have equal and opposite vorticity.

The field curls up into a rotating double loop, as illustrated, because that is a minimum energy configuration. To minimise the energy, as much field as possible should cancel. Any field which cannot cancel should, as 
far as possible, align. Any internal rotational component should align to facilitate this. In the figure, the initial axis of the twist of the photon about the propagation direction as described by equation (5) is aligned perpendicular to that of the momentum flow, with the result that the electric field is everywhere aligned radially. This configuration, with a harmonic rotation about the vertical axis, has maximum magnetic field cancellation as illustrated in figure 3. The radial electric field is required for photon confinement with p-vot, since the new forces act on the electric field component alone. There is also a possibility to produce a dual, magnetic monopole solution if one introduces a q-vot (which acts only on the magnetic field). Such a configuration, which differs in the confined field configuration only by a 90-degree twist around the propagation direction will, presumably, have much higher mass and may, as suggested in earlier work, ${ }^{14}$ be unstable to decay into an electric monopole. That the magnetic monopole is excluded in favour of the electric monopole is also due to the magnetic forces taking priority in the "hierarchy of forces" as discussed by M.B. van der Mark. ${ }^{16}$ It is speculated, however, that this configuration may be the "source" of the weak force within the new theoretical framework.

It is blindingly obvious from figure 3 that, for an outside observer, such vortices exhibit a charge. Also there is an experimental imperative that processes such as that illustrated in figure 2 occur: positronium, consisting of two charged particles decays to uncharged photons. As is observed in experiment, the oscillating electric field in the incident photons has somehow been re-configured (rectified) so that it is everywhere inwards directed (for the electron) or outward-directed (for the positron). Figure 2 provides a picture of how this may occur and figure 3 shows the final configuration of one of the resultant charged vortices. The stability of such a vortex is ensured in that, once it exists, trying to unravel it, or twist it further leads to much higher energy configurations (such as the muon). The only simple way to take an electron to pieces once created is to present it with an anti-vortex, anti-spin, anti p-vot particle - a positron.

This position now enables one to address one of the central mysteries of 20th century physics: just what is charge anyway? Depending on the conventional theoretical basis, charge has two aspects. Within classical field theory, it is the source of the electric field. As viewed from the standpoint of quantum electrodynamics, it is that thing which may emit or absorb photons, the "carrier" of the electromagnetic force. Clearly, physical charge should not be one or the other, but both. The object depicted in figure 3 contains both dynamical p-vot and f-vot. A rest-mass component and a field component. Within the theory, the field is confined by the mass, and the mass by the field. One can view the field component as being like a rapidly rotating light-speed sheepdog, corralling the rest-mass component which is its attractor. Once such objects exist, what is their interaction with field (in the form of light) and p-vot? Free p-vot, on encountering such an object, would simply be absorbed, increasing its energy. If there ever was any such stuff in the locality of the solar system, observations suggest that most of it has long ago been incorporated into existing particles. Light, is another matter. It has been argued above that light is confined into the vortex in the process of pair creation. It follows that such existing vortices have some probability to absorb more light. Equally, the time reversed process, where the vortex sheds light, is allowed provided certain conditions (corresponding to those observed for the physical photon - as argued above) are met. Such vortices provide a still, rest-massive, frame which may emit or absorb photons. They are pitchers and catchers of light. Provided only that the emission or absorption probability is, in dimensionless units, the fine structure constant, this provides the basic starting point for the theory of quantum electro-dynamics. The new solutions then, fulfil both aspects of charge discussed above. They are simultaneously a centre of radial electric field, and that object which may emit or absorb photons. Charge is not a fluid which an electron possesses. Charge results from a stationary flow within a toroidal topology in bi-vector field and momentum space. Charge is a process.

Although it may now be obvious that the new solutions are charged, calculating that charge from first principles is less straightforwards. Assuming that the electron was purely electromagnetic, a lower limit for the electric charge presuming the electromagnetic field was maximally distributed within a rotation horizon defined by the speed of light, remained half and half electric and magnetic and was effectively radial at the characteristic size of the double loop $\left(\lambda_{C} / 4 \pi\right)$ gave a value of $\frac{1}{2 \pi} \sqrt{3 \epsilon_{0} \hbar c}=0.91 q \cdot{ }^{14}$ This is ( perhaps surprisingly) close to the charge observed. As stated, this assumed the electromagnetic energy to be uniformly distributed within a volume of radius $\lambda_{C} / 2$. Imagining that only a quarter of the initial energy manifest as electric field (rather than a half) reduces the lower limit on the charge by a factor of $\frac{1}{\sqrt{2}}$. An upper limit on the electron charge is more difficult to estimate. The average field manifesting radially at the other appropriate length scale in the model, the rotation 
horizon $\lambda_{C} / 2$ would give a charge $\pi^{2}$ larger. Taking another value of the region over which the field is averaged - that of a loop deformed to a single covering disk with radius $\lambda_{C} / 2 \pi$ increases the effective charge by a factor of $\sqrt{\pi^{3}}$. Concentrating the field down to a smaller and smaller radius, of course, raises the effective charge to infinity, as in standard quantum electro-dynamics, and will then require re-normalisation to give sensible results. That the object must be charged is manifest. Calculating that charge depends on the percentage of the initial energy manifesting as electric field, and the precise distribution of that field within the double-covering flow. That distribution should minimise the total energy of the complete solution as well as satisfying the generalised Maxwell equations. Such a particular solution is unlikely to be analytic, but may be subject to finite element modelling. The important thing for the present work is that such solutions are charged, and that the range of charge allowed spans the charge observed.

Another major mystery of physics is how fermions may arise from bosons and just what the fundamental underlying nature of fermions and bosons is anyway. Consider the spin of the double-looped object. Given the momentum of the constituent photon, it is straightforwards to calculate the spin of the photon momentum in the double looped object. ${ }^{14}$ This is just the characteristic length scale of the model $\frac{\lambda_{C}}{4 \pi}$ times the initial photon momentum $\frac{h c}{\lambda_{C}}$. That is the numerical value is half integral: $\frac{\hbar}{2}$. The total spin is then, by symmetry also halfintegral since that of the initial photon rotates, averaging out to zero. By the spin-statistics theorem, therefore, the proposed object is a fermion. More fundamentally, as is clear from figure 3, the object is double-covering over the torus in momentum space and returns to its starting configuration after a 720-degree rotation in the space of an outside observer. That is, the object has the intrinsic symmetry of a fermion. The configuration illustrated in figure 3 is a physical spinor constituted by p-vot (rest mass) and b-vot (electromagnetic) fields. There are four and only four such solutions as the internal spin may twist leftwards ("up" say) or rightwards ("down" say) about the momentum flow and the resultant electric field may be inwards directed (electron) or outwards directed (positron) radially.

A consideration of the internal interference of the fields on overlap leads to a possible physical origin for the exclusion principle itself, as has been discussed in earlier work based on a pure-field model. ${ }^{17}$ Briefly, it was argued there that imposing two parallel spin objects leads to an increase in total energy of the order of the particle masses - a huge repulsive force, whereas overlapping spin antiparallel leads to no increase at all. That the force is of this order agrees with that which has been observed in experiment, both in the observed properties of atoms and in high energy spin polarised scattering. ${ }^{18,19}$ In the present context this argument needs to be updated as the p-vot, which has the S.I. units of the magnetic field, must also be "shared" to give a zero-energy spin antiparallel configuration. The details of how this may work are left to future work.

One further important feature of the earlier model was that it gave a physical explanation of the anomalous magnetic moment of the electron, which could be obtained from a proper consideration of the matching of rotating and non-rotating parts across the rotation horizon. ${ }^{14}$ The value obtained, to first order, was the same as in quantum electrodynamics. That consideration carries over in the present model, as it has the same rotation horizon. Given that the charge, spin and anomalous magnetic moment of the double-looped object are close to those of the electron, it is considered self-evident that, in the theory of vot over 4-dimensional space time forms, it should be be identified with the electron (or positron).

The body of physics, as it stands, is pretty close to the truth in many practical respects. Any new theory should either encompass aspects of the old, improve on it, or provide a proper basis for its starting points. Like its sister model, the Dirac model, the new theory has four and only four rest-massive solutions. The spin up and spin down electron and positron. The differences are twofold - the new model has a (slightly) simpler mathematics and a (slightly) more sophisticated substance. The new mathematics is simpler in that it does not allow the complex imaginary. The substance is more sophisticated in that, in the Dirac model the mass is not brought in as a dynamical term and the fields are brought in through minimal coupling and the vector potential. The substance of the solutions are then a set of "spinor" quantities. In the present theory the substance is just the dynamical root-mass and the physical fields. The four solutions, one of which is illustrated in Fig. 3, are physical spinors constructed of underlying root-mass and fields. Quantum electrodynamics is a theory of photon exchange between charged particles. The new theory provides the physical basis both for the charges and for the exchange particles. This underpins the starting point of the theory and will (hopefully) help removing some 
of the re-normalisation infinities present. The present theory encompasses the Maxwell equations, but provides the physical basis for charge rather than inserting it a-priori.

\section{EXPERIMENTAL TESTS}

Experimental tests of the new theory depend, to a certain extent, on which aspects of it are expressed. For example, it is perfectly possible that the tri-vector term is, as in conventional electromagnetism, precisely zero. This would, however, remove the possibility of describing an underlying angular momentum density, a feature expressed in the experimental properties of particles and markedly absent in conventional electromagnetic theory. Likewise, p-vot (and q-vot) could be absent - the theory then reduces simply to conventional electromagnetism and nothing new will be detected at all.

If the tri-vector term is non-zero, field could be generated from changes in angular momentum, as described by the $\alpha_{i 0}$ term in equation (4). One should then observe electromagnetic radiation from a rapidly stopped neutral, spun, spinning object. This may sound a straightforward enough experiment, but the experimental challenge will lie in finding uniformly uncharged material (even the neutron has a very large magnetic moment for such a small particle), finding a suitable means of stopping it spinning (note that hitting it with photons or electrons may impart angular momentum as well), and removing the possibility of the object simply absorbing its own radiation as low-energy heat. One possibility may be to generate one component of field, but not the other, so that it cannot propagate away or be re-absorbed. Such considerations may help elucidate some peculiar experimental effects such as the Aspden effect. ${ }^{20}$

As mentioned above, free p-vot (if it exists) is strongly scavenged by existing material particles. Such a process should manifest at low densities as an increase in low energy heat. If there are yet regions of the universe where free p-vot exists, it will manifest itself in particular properties. Firstly, even at very low densities, it will interact gravitationally, making it a candidate for dark matter. Secondly, it will heat matter impinging on it -which may be detectable. Thirdly, if there were to exist regions of very high p-vot density, matter incident on it would heat very rapidly, perhaps yielding $\mathrm{x}$ or gamma ray radiation. A re-analysis of existing cosmological data may then be sufficient to confirm or deny the existence of free p-vot.

It may be possible to generate free p-vot in the lab, for example by cancelling electromagnetic fields in the vacuum, where the $\mathrm{p}$-vot is then formed to conserve energy. One could then attempt to probe this region with particle beams or low-energy photons. Plane polarised photons with a (half) wavelength similar to the region of p-vot, for example, should then be deflected in the electric-field plane, depending on the phase of the field. In designing experiment it should be noted that free p-vot will fall in a gravitational field. This effect is then best measured on a wall-mounted light table.

It is quite possible, indeed likely, that p-vot exits only as a transient dynamical effect in existing rest-massive particles. In this case the most direct tests of the new theory lie in high energy polarised particle scattering. Polarised electrons, for example, incident on electrons within a strong magnetic field, should show asymmetric, non-point-like scattering. Again, a re-analysis of existing experimental data through the prism of the new theory may confirm or deny this possibility.

\section{CONCLUSIONS}

A theory has been developed of root-energy (vot) transforming within sixteen linearly independent relativistic space time forms derived from the four dimensions of space and time. Root-energy in each of these forms is then identified with physically observed phenomena. Integration over the p-vot squared is an element of rest mass-energy. Following Maxwell, the vector part is identified directly with the (root) charge and current density. This is zero in the rest-massless case of the photon, but non-zero for rest-massive solutions such as the electron and positron, leading to a non-zero charge. The six bi-vector components are identified with the physical electromagnetic field. This is oscillatory for the photon, but uni-directional (radial) for the electron and positron The tri-vector is identified with a root angular momentum density - allowing an integration of underlying spin into the theory of electromagnetism. The quadri-vector is identified with an inward or outward directed four-volume root-energy, whose derivative yields angular momentum components. The interaction between the various space-time forms of vot is then argued to be responsible for generating the forces required for the system 
as a whole to confine itself into particles. Possible further developments of the theory to describe these forces explicitly have been proposed. Within the new theory a new kind of solution, equation (5) has been proposed satisfying the (field only) Maxwell equations. This solution has energy proportional to frequency, a fixed angular momentum limit and is identified with the physical photon. Extending the Maxwell equations by introducing a further rest-mass component has allowed qualitatively new kinds of solutions containing, as well as the rest-mass component, re-circulating field components. Such solutions are necessarily charged, have half-integral spin and have the proper 720 degree symmetry of fermions. These act as a possible centre for the emission and absorption of photons. These solutions are identified with the electron and positron. Possible routes for the experimental testing of the new theory have been proposed.

\subsection{Acknowledgments}

This work flows from a long and fruitful collaboration with my friend and co-pirate Martin van der Mark. I am grateful to Phil Butler for his education in algebra, to Stephen Leary for his valuable contribution, to John Duffield, Tim Drysdale, Richard Gauthier and David Mathes for useful suggestions and to Nick Bailey, Nick Green and John Weaver for their constant encouragement. The figures were drawn using Povray. Preprint of article : Proc. SPIE 9570, The Nature of Light: What are Photons? VI, 957015 (September 10, 2015); doi:10.1117/12.2188259. Terms of Use: http://spiedigitallibrary.org/ss/TermsOfUse.aspx

\section{REFERENCES}

[1] Williamson J. G. and Leary S.J., "Absolute relativity and the quantisation of light", SPIE Optics + Photonics, San Diego, 9570-41 (9-13 August 2015).

[2] Williamson J. G., "A new theory of light and matter". FFP14, Marseille, France, (2014).

[3] Moon, P. and Spencer D.E.,[Field theory for engineers], van Nostrand, (1961).

[4] Lounesto, P., [Clifford Algebras and Spinors], Lond. Math. Soc. Lecture Notes Series 239, (1997).

[5] Hestenes, D., "Zitterbewegung in Quantum Mechanics", Found Phys, 40 (2010).

[6] Penrose, R., [The Road to Reality], Jonathan Cape (2004).

[7] Wittgenstein, L., [Tractatus Logico-Philosophicus], (1921), www.gutenberg.org/etext/5740.

[8] Williamson J. G., "On the nature of the electron and other particles", paper presented at The Cybernetics Society 40th Anniversary Conference (2008).

[9] Williamson,J.G. et. al, Phys. Rev. B 42, 7675, 1990.

[10] Maxwell, J.C., [A treatise on electricity and magnetism]. 3rd edition, Dover(1954).

[11] Mead, Carver A., [Collective electrodynamics], MIT press, (2002).

[12] Leary,S.J. "Investigation of electromagnetism in a real Dirac algebra". PhD Thesis, University of Glasgow, (2007).

[13] Dirac, P. A. M., [The Principles of Quantum Mechanics], (Oxford U.P., London, 1958). 4th ed.

[14] Williamson, J.G. \& van der Mark, M.B., "Is the electron a photon with toroidal topology?", Ann. Fondation L. de Broglie 22, 133 (1997).

[15] Waite,T., "The Relativistic Helmholtz Theorem and Solitons". Phys. Essays, 8, 60 (1995).

[16] M.B. van der Mark, "On the nature of "stuff" and the hierarchy of forces", SPIE Optics + Photonics, San Diego, 9570-53 (9-13 August 2015).

[17] Williamson J. G., "Fermions from Bosons and the origin of the exclusion principle", Proceedings of MENDEL $2012(2012)$

[18] O'Fallon et al., Phys. Rev. Lett. 39, 733, (1977).

[19] Krisch, A.D., "The spin of the proton", Scient. American. (May, 1979).

[20] Aspden, H, "Discovery of virtual inertia". New energy news. Volume 2, Number 10, (Feb, 1995). 